http://jmscr.igmpublication.org/home/ ISSN (e)-2347-176x ISSN (p) 2455-0450 crossref DOI: https://dx.doi.org/10.18535/jmscr/v9i6.14

\title{
Goldenhar Syndrome (Oculo-Auriculo-Vertebral Syndrome) - A Rare Case Report
}

\author{
Authors \\ Dr Manvi Katoch ${ }^{1}$, Dr Shiwani Thakur ${ }^{2 *}$, Dr Tanvi Katoch ${ }^{3}$ \\ ${ }^{1}$ Anaesthesiologist, Zonal Hospital, Dharamshala, H.P. \\ ${ }^{2}$ Senior Resident, Department of Pediatrics, JLNMCH Bhagalpur, Bihar \\ ${ }^{3}$ Senior Resident, Department of Obstetrics and Gynecology, PGI, Chandigarh \\ *Corresponding Author \\ Dr Shiwani Thakur \\ Senior Resident, Department of Pediatrics, JLNMCH Bhagalpur, Bihar, India
}

\begin{abstract}
Goldenhar syndrome is a rare congenital defect involving first and second branchial arches and is characterized by incomplete development of nose, ear, palate, eyes, mandible and lip. It is also known as oculo-auriculo-vertebral syndrome. Reconstructive surgery is done to correct the facial defects and other structural abnormalities.
\end{abstract}

Keywords: Brachial arch, Ear tags, Goldenhar syndrome, Hypoplasia, Oculo-auriculo-vertebral syndrome.

\section{Introduction}

Goldenhar syndrome is a rare defect characterized by defect in morphogenesis of the first and second branchial arches. This condition mainly affects oral cavity, ears, eyes and vertebrae. Goldenhar syndrome is also known as oculo-auriculovertebral syndrome. It is a variant of hemifacial microsomia. Frequency of occurrence is estimated to be 1 in 3,500 to 1 in 5,600and there is a slight male predominance(3:2). ${ }^{[1]}$ Herein, we present a case of Goldenhar syndrome focussing on its etiology and characteristic clinical features.

\section{Case Report}

A 2days old male newborn baby was admitted in the pediatrics department with the complaint of delayed cry after birth (after $10 \mathrm{~min}$ ) after full term normal vaginal delivery in a private hospital.
There was no history of prolonged labour in mother. There was history of one episode of seizure in baby, which was of subtle type. There was no history of cyanosis and frothing from mouth, loss of consciousness in the child as per his mother.

On head to toe examination-anterior fontanelle was open and full. There was facial hypoplasia and hypoplasia of mandibular region (micrognathia) [Figure 2]. Preauricular ear tags in right ear [Figure 1] and microtia of left ear [Figure 2, Figure 3] were detected. Cleft palate was also found on oral examination. On auscultation, there was bilateral equal air entry and a pansystolic murmur of grade II was heard in left parasternal area. On CNS examination, neonatal reflexes were poor and baby was lethargic. There was no history of any drug intake (except iron and folic acid), 
diabetes, hypertension and hypothyroidism or hyperthyroidism in mother during pregnancy. There was no history of similar complaint or features in sibling or family.

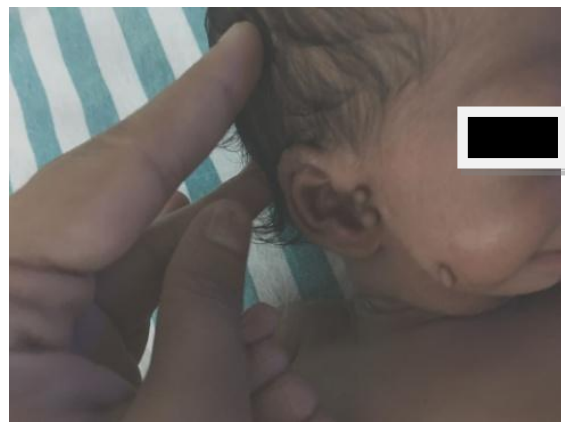

Figure 1: Preauricular ear tags in right ear

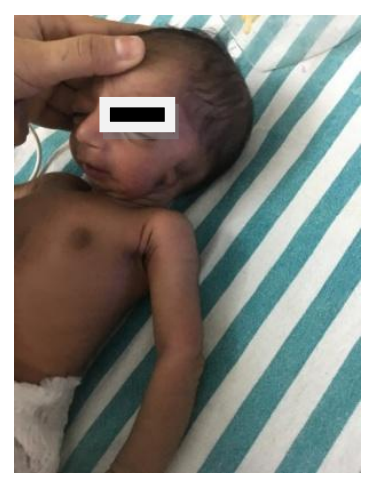

Figure 2: Facial micrognathia with microtia of left ear

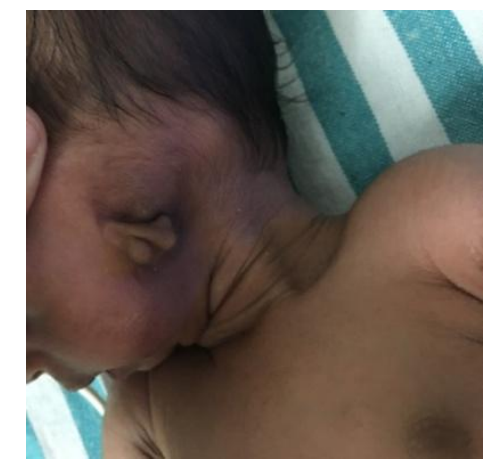

Figure 3: Microtia of left ear

Investigations were as follows - random blood sugar was $100 \mathrm{mg} / \mathrm{dl}$, hemoglobin was $14 \mathrm{~g} / \mathrm{dl}$, serum electrolytes were in normal limits, Neurosonography was found to be normal and sleep EEG showed normal activity. X-ray spine showed hemivertebrae in cervical region. Echocardiography presented moderate type ventricular septal defect in heart. Thereafter, diagnosis of Goldenhar syndrome was made.
Child was then referred to paediatric surgery department for further follow up needed for reconstruction of deformed ear and correction of other facial defects. Parents were counselled about the child's condition.

\section{Discussion}

Goldenhar syndrome is a rare congenital defect. Goldenhar syndrome is also known as oculoauriculo-vertebral syndrome. It was first time observed by Carl Ferdinand Von Arlt. ${ }^{[2]}$ However, the syndrome was first described more clearly by Maurice Goldenhar, and thus, this syndrome was named after him. ${ }^{[3]}$ Goldenhar syndrome is characterized by facial anomalies along with epibulbar dermoid, accessory preauricular appendages and vertebral anomalies. ${ }^{[4,5]}$ There is malformation in the morphogenesis of first and second branchial arches. The condition can be inherited in an autosomal dominant or sporadic manner. It can occur more frequently in one member of a monozygotic twin pair and has been seen in cases following assisted reproductive techniques. Anomalies tend to be asymmetric and $70 \%$ unilateral. When unilateral, it tends to be right sided. ${ }^{[6]}$

Exact etiology is not known clearly. However, maternal diabetes has been associated in some cases. Ingestion of certain drugs like thalidomide, retinoic acid, tamoxifen, and cocaine by mothers during pregnancy increases the risk of this syndrome in the newborn. This disorder is believed to be due to interference with embryonic vascular supply and focal hemorrhage in the developing first and second branchial arch. ${ }^{[7]}$

Classical clinical findings in Goldenhar syndrome can include the following-

- Face- Hypoplasia of malar, maxillary, mandibular region and facial musculature, micrognathia, macrostomia.

- Ear- Microtia, accessory preauricular ear tags most commonly in a line from tragus to the corner of the mouth, middle ear and inner ear anomalies. 
- Eye- Epibulbar dermoid, lipodermoid, strabismus.

- Oral- Cleft lip, cleft palate, tongue anomalies.

- Vertebral- Hemivertebrae or hypoplasia of vertebrae mostly cervical. ${ }^{[4,5]}$

- Cardiac- ventricular septal defect (VSD), atrial septal defect (ASD), patent ductus arteriosus (PDA), tetralogy of fallot (TOF) can be seen.

- Central nervous system- Hydrocephalus, intracranial dermoid cyst, agenesis of corpus callosum, calcification of falx cerebri, lipoma.

- Genitourinary- Ectopic or fused kidneys, renal agenesis, multicystic kidneys, vesicoureteral reflux.

- Other systems- Speech delay, laryngeal anomaly, esophageal atresia, rib anomalies etc. Most of these patients are of normal intelligence. Intellectual disability can be seen in $13 \%$ of patients. ${ }^{[5]}$

Diagnosisis mainly based on clinical presentation. Other differential diagnosis for similar presentation are Townes-Brocks syndrome and Treacher-Collins syndrome. Townes-Brocks syndrome has renal anomalies and anal anomalies in addition to facial anomalies which were not seen in this case. Treacher-Collins syndrome show bilateral involvement of similar anomalies, and so, it was ruled out in our case. ${ }^{[2]}$ Reconstructive surgery is the mainstay treatment in Goldenhar syndrome. Facial anomalies and associated structural deformities are corrected by multiple plastic surgeries. A few patients require assistance by means of hearing aids and glasses. Stem cell grafting has been used to reprogram eye dermoids, halting the regrowth of eye dermoids. Prognosis is usually good in patients with no systemic complications. $^{[8]}$

\section{Conclusion}

Though Goldenhar Syndrome is rare defect, it can cause severe systemic complications if not detected early. Timely diagnosis and management is crucial. Patients with this defect usually suffer from psychological problems in later stages of life. Proper counselling should be done in intervals for such patients to lead a quality life.

\section{References}

1. Grabb WC. The first and second brachial arch syndrome. Plast Reconstruct Surg 1965;36:485-508.

2. Gorlin RJ, Cohen MM, Levin LS. Syndromes of the Head and Neck. New York: Oxford University Press; 1990. p.707-8.

3. Mellor DH, Richardson JE, Douglas DM. Goldenhar's syndrome. Oculoauriculo vertebral dysplasia. Arch Dis Child 1973;48:537- 41.

4. Bekibele CO, Ademola SA, AmanorBoadu SD, Akang EE, Ojemakinde KO. Goldenhar syndrome: A case report and literature review. West Afr J Med 2005;24:77-80.

5. Kokavec R. Goldenhar syndrome with various clinical manifestations. Cleft Palate Craniofac J 2006;43:628- 34.

6. Ashokan CS, Sreenivasan A, Saraswathy GK. Goldenhar syndrome - Review with case series. J Clin Diagn Res 2014;8:ZD17-9.

7. McKenzie J. The first arch syndrome. Arch Dis Childh 1958;33:477-486.

8. Mehta B, Nayak C, Savant S, Amladi S. Goldenhar syndrome with unusual features. Indian J Dermatol Venereol Leprol 2008;74:254-6. 